\title{
ZUR PHRASEMVERMITTLUNG IM DAF-UNTERRICHT
}

\author{
Doc. dr. Diana Babušytè \\ Mykolas Romeris Universität
}

\begin{abstract}
Annotation. In der Fremdsprachendidaktik und in den gängigen DaF-Lehrwerken wird dem Phrasemerwerb wenig Aufmerksamkeit geschenkt. Aus diesem Grund ist das selbständige Aufarbeiten von kreativen Unterrichtsmaterialen der Lehrkräfte gefragt. Der vorliegende Beitrag versteht sich als ein Plädoyer für den systematischen Ansatz von Phraseologie im DaF-Unterricht auf verschiedenen Niveaustufen. Im Mittelpunkt des Beitrags stehen sowohl einschlägige Forschungsergebnisse der phraseologischen Diskussionen sowie die Ansätze der Phrasemvermittlung in den geläufigen DaF-Lehrwerken. Damit wird das Ziel verfolgt, eine theoretisch fundierte Beschreibung von Lernprozessen zur Förderung phraseologischer Kompetenz zu geben sowie darauf aufbauende Didaktisierungsvorschläge zur Phrasemvermittlung zu präsentieren.
\end{abstract}

Schlüsselwörter: Phraseologie, Phraseme, Phrasemvermittlung, phraseologische Kompetenz

\section{Einleitende Bemerkungen}

Phraseme sind ein fester Bestandteil jeder Sprache, ohne deren Beherrschung die Kommunikation in einer Fremdsprache kaum möglich ist (Fleischer 1997: 32; Alenius 2014: 17). Als Teil eines kulturellen Erbes sind sie im Gedächtnis eines Muttersprachlers gespeichert, verschönern sowohl die geschriebene als auch die gesprochene Sprache und machen sie lebendiger. Aus diesem Grund halte ich es für ausgesprochen wichtig und notwendig, Lernende für deren Gebrauch zu sensibilisieren.

Obwohl die Kenntnis phraseologischer Sprachmittel zu einer wichtigen Komponente der kommunikativen Kompetenz gehört, wird sie in fremdsprachendidaktischen Diskussionen und in den gängigen Lehrwerken häufig unterschätzt. Darüber hinaus sind die didaktischen Konzepte der Einbindung von Phrasemen in den DaF-Unterricht wenig diskutiert und erforscht (Ehrhardt 2014: 1). Die Bekanntschaft mit phraseologischen Ausdrücken ist aber für jeden DaF-Lerner unerlässlich, denn wer sich auf das Abenteuer mit der deutschen Sprache einlässt, sollte schon möglichst früh lernen, mit Phrasemen umzugehen, d.h. sie entdecken, ver- 
stehen und verwenden. Deswegen sollten solchen sprachlichen Phänomene sowohl im DaF-Unterricht als auch in der diesbezüglichen Methodik und Didaktik stärker berücksichtigt werden.

Ich bin der Meinung, dass die phraseologische Kompetenz als eine wesentliche Bedingung der Sprachbeherrschung betrachtet werden soll und stimme Hallsteinsdóttir (2011: 5) und Hessky (1997: 139) zu, die großen Wert auf den Erwerb von Phrasemen bei der Förderung der Sprachkompetenz legen: „Je mehr Phraseme ein Fremdsprachler kennt, desto besser ist seine fremdsprachliche Sprachkompetenz". In der Tat ist die norm- und situationsgerechte Anwendung der Phraseologismen bzw. Phraseme ein Zeichen für ein hohes Sprachniveau. Deshalb soll die Vermittlung von phraseologischen Ausdrücken im DaF-Unterricht an Bedeutung gewinnen, denn ihre aktive Beherrschung kann den Lernenden in der rezeptiven und produktiven Kompetenz mehr Sicherheit verleihen und sie sprachlich weiter bringen (Gündoğdu 2007: 12).

Im vorliegenden Beitrag wird für eine stärkere Einbindung der Auseinandersetzung mit Phraseologie in den DaF-Unterricht plädiert. Es werden Didaktisierungsvorschläge für die Realisierung dieses Anliegens präsentiert bzw. die wichtigsten Strategien für eine positive Vermittlung von Phrasemen im DaF-Unterricht erläutert. Zuerst werden einschlägige Forschungsergebnisse der phraseologischen Diskussionen vorgestellt, und es wird ein Überblick über den Ansatz der Phrasemvermittlung in den geläufigen Lehrwerken gegeben. Im Weiteren wird ein Didaktisierungsvorschlag zur Phrasemvermittlung präsentiert und darüber diskutiert, welche Schritte für einen erfolgreichen Ansatz der Phraseme im DaF-Unterricht angewandt werden könnten.

\section{Phraseodidaktik}

Die phraseodidaktische Forschung ist im Grunde genommen relativ jung, denn sie hat erst eine dreißigjährige Tradition. Auf dem Gebiet der Fremdsprachendidaktik wurde bis in die 90er Jahre selten und sehr kurz auf die Bedeutung der phraseologischen Kompetenz und auf Lernschwierigkeiten beim Erwerb phraseologischer Einheiten hingewiesen (vgl. Fleischer, 1997: 6f). Die Frage, welche Methoden und Übungsformen für die Vermittlung der Phraseme im Fremdsprachenunterricht sinnvoll sind, konnte man lange nicht beantworten. Diesbezüglich hat Kühn (1992) diesen Forschungsbereich als "phraseodidaktischen Dornröschenschlaf“ bezeichnet und Wotjak (1996) behauptete, dass Phraseodidaktik über lange Zeit hinweg ein „Stiefkind der Sprachdidaktik“ gewesen war. Erst in den 90er Jahren erscheinen in den phraseodidaktischen Diskussionen mehr Arbeiten und Aufsätze, die phraseodidaktische und methodische Fragestellungen ausführlich darstellen (Kühn, 1992; Lüger, 1997). Der Fachbereich erfreut sich heute eines gewissen Interesses und ist relativ gut erforscht, was vor allem mit einem wachsenden Interesse der Lehrkräfte und Lernenden an der Vermittlung der Phraseologie im Sprachunterricht 
zusammenhängen kann (Valenčič Arh 2014: 368).

Die erste didaktisch praktikable Vermittlungsform der Phraseme im DaFUnterricht stammt von Kühn (1992) und seinem ,phraseodidaktischen Dreischritt“: entdecken, erkennen und verwenden, der als eines der wichtigsten Lernverfahren beim Erlernen fremdsprachlicher Phraseme zu betrachten ist und sich auf beliebigen Sprachniveaus und mit unterschiedlichen Lernergruppen umsetzen lässt (vgl. Gündoğdu 2007: 14). Das war der erste Versuch die Frage zu beantworten, wie phraseologische Einheiten im Sprachunterricht vermittelt, bearbeitet, gelernt und gebraucht werden sollten. Die Grundlage für dieses Verfahren schöpfte Kühn aus seinen praktischen Unterrichtserfahrungen. Lüger (1997) vervollständigte diese Methode, indem die zusätzliche Phase „festigen“ zwischen die zweite (,erkennen“) und die dritte (,verwenden“) Phase eingeschoben wurde, weswegen heute die Rede vom ,phraseodidaktischen Vierschritt“ ist.

\section{„Phraseodidaktischer Drei- bzw. Vierschritt““}

Bevor der phraseologische Dreischritt genauer behandelt wird, muss ein wichtiger Aspekt der Vermittlung von Phrasemen im DaF-Unterricht besprochen werden, u.z. die richtige Wahl des Unterrichtsmaterials. Für die Vermittlung phraseologischer Ausdrücke sollten aktuelle, im alltäglichen Sprachgebrauch oft gebräuchliche Phraseme behandelt werden. Deshalb sind dafür authentische Texte geeignet, die eine didaktisch-methodische Hilfe zur Phrasemauswahl anbieten sowie zur Erweiterung des Wortschatzes und zur Entwicklung kommunikativer Kompetenz der Lernenden beitragen.

In den geläufigen DaF-Lehrwerken werden Phraseme meistens als isolierte und kontextfreie Beispielsätze abgehandelt. Ein solches Verfahren erscheint wenig sinnvoll, weil diese Art von Aufgaben nur auf das kontextfreie Auswendiglernen hinzielt und keinesfalls die Entwicklung der kommunikativen bzw. phraseologischen Kompetenz berücksichtigt. Das hat Kühn dazu bewogen, seine eigene produktive Methode zu entwickeln, die ausschließlich authentische Texte als Grundlage voraussetzt. Bei der Arbeit mit authentischen Texten liegt der Schwerpunkt nicht nur darin, dass die Lernenden Phraseme entdecken und sie entschlüsseln bzw. verstehen, sondern sie sollen auch lernen, ihre Bedeutung zu festigen und sie schließlich selbstständig im kommunikativen Sprachgebrauch zu verwenden.

\section{Erster Schritt: Entdecken}

Der erste Schritt besagt, dass die Lernenden dafür sensibilisiert werden sollten, ob Phraseme im Text vorkommen oder nicht, d.h. sie sollten fähig sein, phraseologische Ausdrücke von anderen sprachlichen Strukturen zu unterscheiden. Deswegen ist es wichtig, die Lernenden auf die besondere Struktur bzw. besondere Merkmale der Phraseme aufmerksam zu machen sowie ihre Typen im engeren und weiteren 
Sinne (z.B. Sprichwörter, Idiome, Kinegramme, Zwillingsformeln, Kollokationen etc.) vorzustellen, damit sie diese sprachlichen Phänomene von den freien Wortverbindungen unterscheiden können. Den Lernenden soll zum Beispiel erklärt werden, dass Phraseme wörtlich oft keinen Sinn machen und sich durch morphosyntaktische Besonderheiten auszeichnen können. Man kann beispielsweise auf den fehlenden Artikel hinweisen (Schwein haben vs. ein Schwein haben, Hahn im Korb vs. ein Hahn im Korb), auf irreguläre Flexionsbesonderheiten (lieb Kind machen, statt. liebes Kind machen) oder auf einige leicht erkennbare Strukturen wie komparative Phraseologismen (stumm wie ein Fisch, scheu wie ein Reh) und Zwillingsformeln (Arm in Arm, Schulter an Schulter, von Fall zu Fall, nach und nach) hinweisen.

\section{Zweiter Schritt: Entschlüsseln}

Nachdem die Lernenden Phraseme in einem Text entdeckt haben, folgt die nächste Phase, in der sie sie entschlüsseln sollen. Dies kann auf verschiedene Weisen durchgeführt werden, wie z.B. durch ein mentales Bild. Laut Kühn (1992: 179) ist dieses Verfahren etwas problematisch, denn Phraseme können fehlerhaft interpretiert werden, besonders wenn sie ähnliche oder vollkommen andere Bildentsprechungen in der Muttersprache der Lernenden haben. Darüber hinaus soll auch darauf hingewiesen werden, dass nicht alle Phraseme bildlich vorstellbar sind. In solchen Fällen können Wörterbücher benutzt werden, die aber im Unterricht nicht immer zur Verfügung stehen. Aus diesem Grund sollte den Lernenden der Weg gezeigt werden, wie die Bedeutung eines Phrasems durch den Kontext selbstständig entschlüsselt werden kann.

Für diesen Zweck sind authentische Texte (z.B. Horoskope, Fimankündigungen, Zeitungsartikel etc.) geeignet. Nach dem Lesen eines Textes werden Übungen gemacht, die den gerade gelesenen Text behandeln und dabei helfen, die Bedeutung der Phraseme aus dem Kontext zu intepretieren. Obwohl die Arbeit mit solchen kontextbezogenen Bedeutungsinterpretationen sehr zeitaufwändig ist, ist es trotzdem der beste Weg für die Lernenden, Phraseme zu verstehen. Eine zusätzliche Möglichkeit für die Bedeutungserschließung eines Phrasems stellen Bilder dar, deren Ansatz nicht immer erfolgreich verlaufen kann, da, wie schon vorher erwähnt wurde, viele Phraseme bildlos sind. Hier muss aber aus der Erfahrung betont werden, dass die Arbeit mit Bildern eine der bevorzugten Übungsformen bei den Lernenden ist.

\section{Dritter (vierter) Schritt: Festigen und Verwenden}

Lüger (1997: 102) erweitert in seiner Übungstypologie Kühns Dreischritt und fügt zwischen die zweite und die dritte Phase den Schritt „festigen“ hinzu. Dazu gehören die auf einen produktiven Gebrauch abzielenden Arbeitsschritte in Form von zahlreichen Übungen, die auf das Festigen der besonderen Merkmale von 
Phrasemen wie beispielsweise von morphosyntaktischen Strukturen und stabilen lexikalischen Komponenten mit ihren variablen Formen hinzielen (Zuordnungs-, Ergänzungs- Multiple-Choice-Übungen).

Nach dem Festigen eines Phrasems in seiner Form und Bedeutung folgt die vierte und die schwierigste Phase: Verwenden und Gebrauch der Phraseme, d.h. die Lernenden sollten lernen, sie situationsangemessen zu verwenden. Hier ist das Einbeziehen der Phraseme auf nachvollziehbare Situationen und Kontexte erforderlich, weil die Lernenden mit der jeweiligen Situation vertraut sein müssen, um sie korrekt verwenden zu können. Die strikte Kontextualisierung von allen Übungen spielt hier eine entscheidende Rolle, denn die Produktionsaufgabe sollte in demselben Rahmen wie der Text erfolgen, aus dem die Entschlüsselung der Phraseme gemacht wurde. Wenn der Text beispielsweise eine Buchankündigung war, dann sollte es sich bei der Produktionsaufgabe um eine ähnliche Textsorte handeln. Erst später, in höheren Lernstufen, kann dieser Schritt auf andere Situationen übertragen werden.

Zusammenfassend lässt sich sagen, dass der phraseologische Drei- bzw. Vierschritt die am meisten praktikable und ganzheitliche Methode zur systematischen Behandlung der Phraseme im DaF-Unterricht ist, deren Vorteil in der Erweiterung des Wortschatzes sowie dem Kennenlernen von Möglichkeiten und Verfahren des selbständigen Weiterlernens liegt.

\section{Phraseme in DaF-Lehrwerken}

Phraseme können in Form von verschiedenen Übungen und Aufgaben didaktisiert und vermittelt werden, wie z.B. Übungen zur Sensibilisierung, formbezogene Übungen, bedeutungsbezogene Aufgaben, Übungen zur Textproduktion etc. Dabei können sie verschiedene Formen haben, wie z.B. Ergänzungs-, Einsetzungsübungen, Kombinationsübungen, Korrekturaufgaben, Korrektur falscher Bedeutungsangaben, Zuordnung von Synonymen etc. Eine große Bedeutung bei diesen Übungen hat das bildliche Material: Bilder, Karikaturen, Comics usw. Im Folgenden werden Beispiele zur Phrasemvermittlung in den gängigen Lehrwerken präsentiert, und es wird veranschaulicht, in welcher Form nun phraseologische Ausdrücke in den Lehrwerken vorkommen.

In den Grundstufenlehrwerken wird sich größtenteils auf die Vermittlung von Kollokationen und Grußformeln (Phraseologie im weiteren Sinne) konzentriert, die selbstverständlich in jedem Lehrwerk vorhanden sind und meistens durch Bilder, Dialoge, Ergänzungsübungen sowie durch andere Typen von Aufgaben didaktisiert werden (sieh Abb. 1). Idiomatische Redewendungen und Sprichwörter (Phraseologie im engeren Sinne) werden eher außer Acht gelassen. 


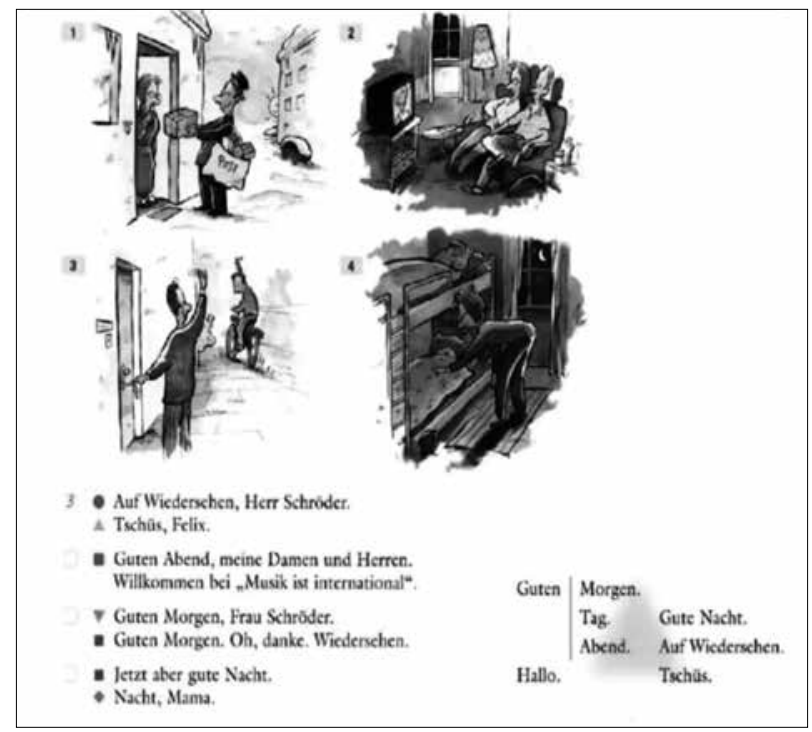

Abb. 1: Schritte international neu 1 (S. 12);

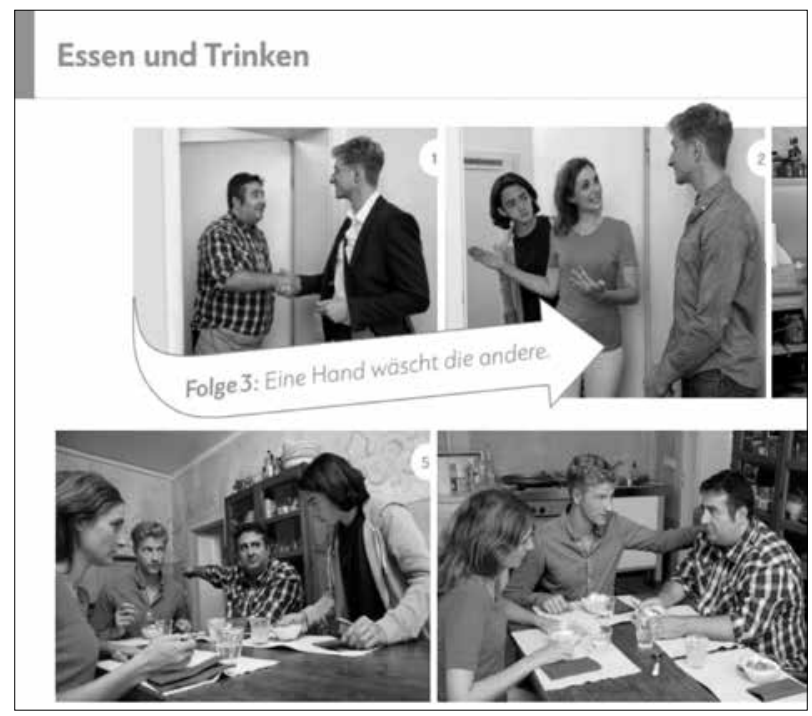

Abb. 2: Schritte international neu 3 (S. 34)

Aus diesem Grund ist es sehr erfreulich, dass Phraseme in dem Grundstufenlehrwerk Schritte international neu als Einstieg in ein Thema eingesetzt werden. Jede Lektion beginnt mit einer Fotohörgeschichte (auch mit Videoclips), deren Titel ein Sprichwort oder eine Redewendung darstellt (sieh Abb. 2). Dass ein Grundstufenlehrwerk Phraseme in den Mittelpunkt seines Konzeptes stellt, ist aber eher eine Seltenheit. 
Im Weiteren wird eine Aufgabe aus der Reihe Schritte international (Band 2) präsentiert, die explizit auf die Vermittlung der Phraseme hinzielt (sieh Abb. 3). Hier geht es um Redewendungen und Sprichwörter mit der Komponente „Körperteil" (Somatismen), die mithilfe von Bildern und Sätzen, in die diese Redewendungen integriert sind, zuerst entschlüsselt und dann einander zugeordnet werden sollen. Danach folgt eine Produktionsaufgabe, in der kurze Gespräche mit den Redewendungen gebildet werden sollen.

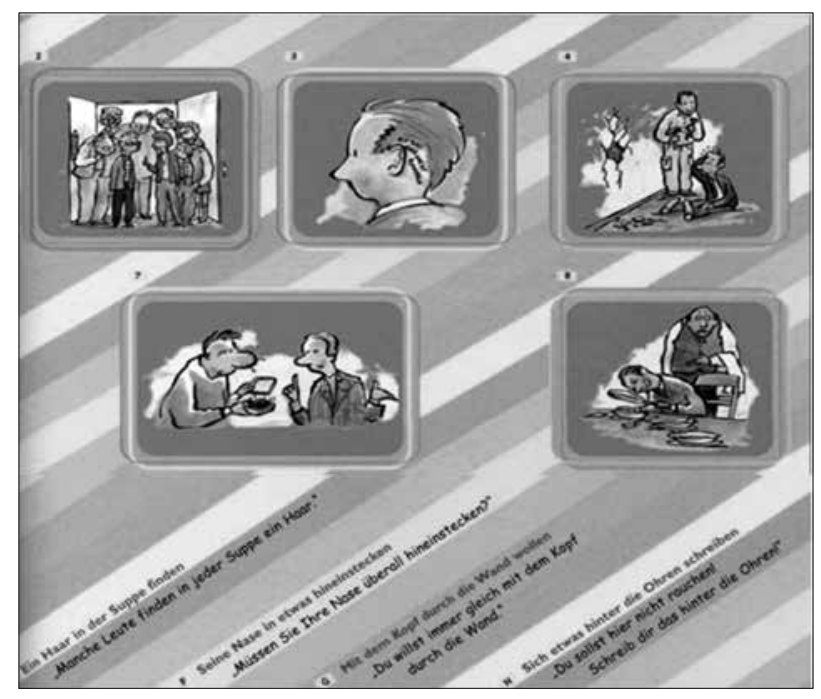

Abb. 3: Schritte international 2 (S. 36-37);

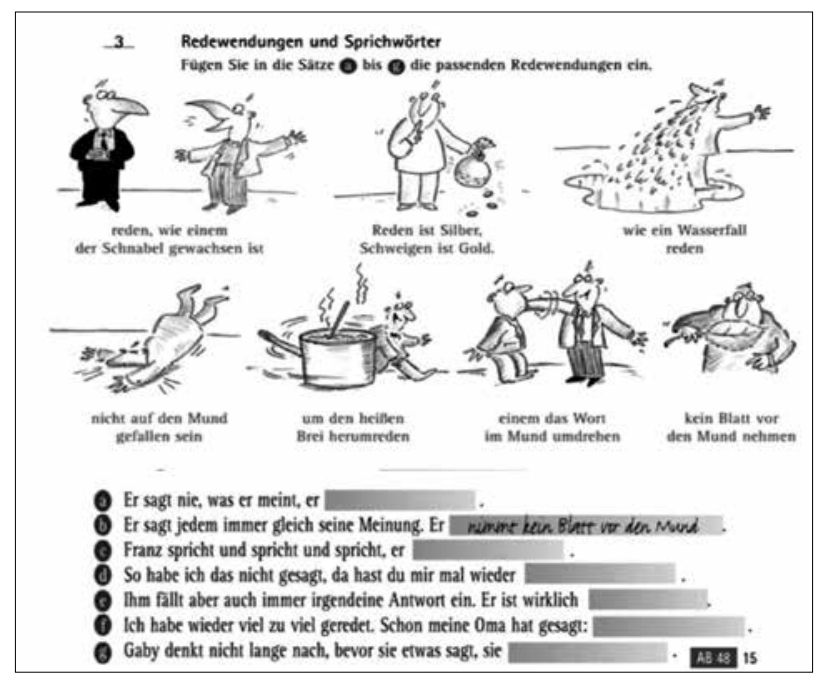

Abb. 4: em Abschlusskurs (S. 53) 
In den Lehrwerken für Fortgeschrittene wird mit Sprichwörtern und anderen phraseologischen Ausdrücken viel komplexer gearbeitet. Das nächste Beispiel bezieht sich auf die Arbeit mit Redewendungen im Lehrwerk „em“ (Abschlusskurs). Hier wird auf die metaphorische Bedeutung der Wendungen eingegangen, die durch Bilder zum Ausdruck gebracht wird. Dabei werden die Wendungen je nach dem Sinn in den Kontext eingebunden (sieh Abb. 4).

In der nächsten Übung (sieh Abb. 5) handelt es sich um eine Zuordnungsaufgabe, in der die Bedeutung von Redewendungen erklärt wird. Solche Aufgaben werden häufig von Bilddarstellungen abgeleitet.
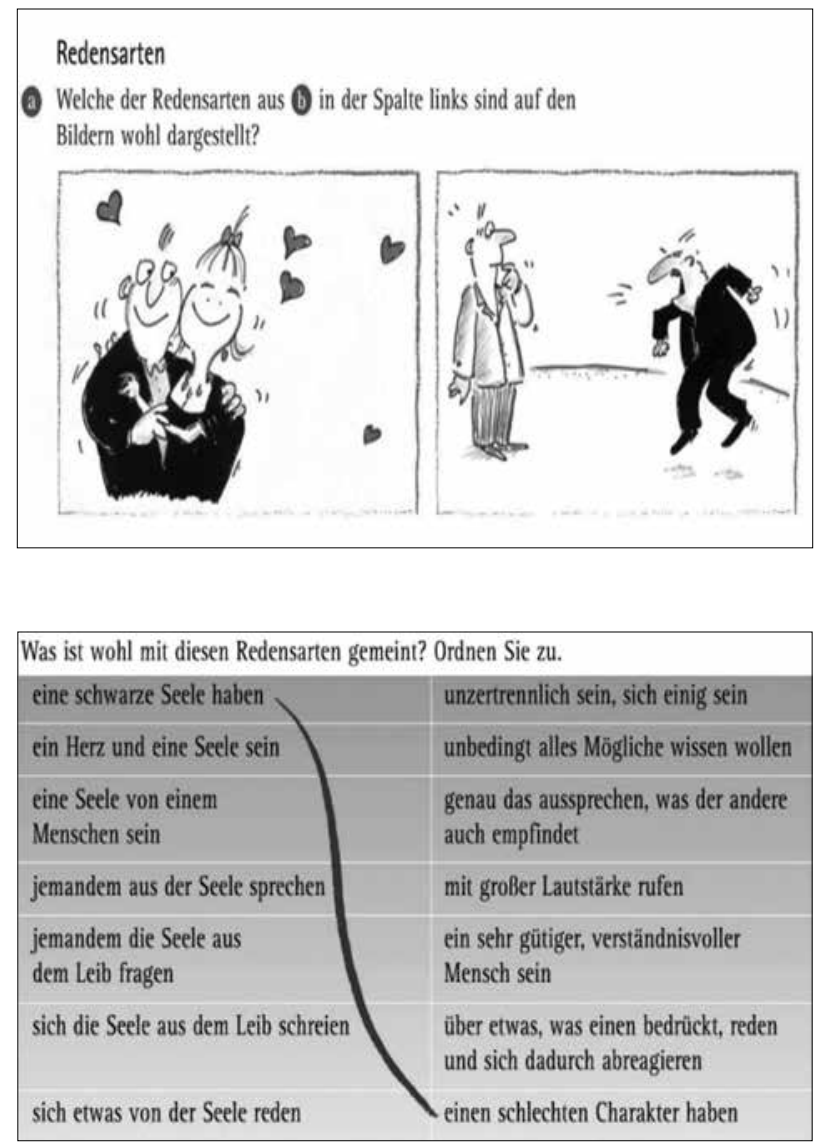

Abb. 5: em neu Abschlusskurs (S. 61)

Da phraseologische Materialien in den allgemeinen DaF-Lehrwerken immer noch unterrepräsentiert sind, ist das selbständige Aufbereiten von gut geeigneten Übungen erforderlich. Um den zeitlichen Rahmen nicht zu sprengen und die Kompetenz der Lernenden nicht zu überfordern, ist viel Vorbereitung seitens der Lehrkraft vonnöten. Die Lehrmaterialien sollten Interesse und Motivation für die weitere Beschäftigung mit diesem Phänomen fördern. Besonders schwierig gilt das für 
die Grundstufe, wo Phraseme viele Schwierigkeiten bei ihrer Entschlüsselung und Verwendung bereiten.

Erwähnenswert und vorbildlich ist das umfangreiche multimediale Unterrichtsmaterial zur Phraseologievermittlung auf der Internetseite http://frazeologie. ujepurkyne.com, das 2006 im Rahmen eines vom tschechischen Schulministerium geförderten Projektes fertig gestellt wurde (Bergerova 2011: 108). Das Lern- und Lehrmaerial ist dreiteilig: In den ersten zwei Teilen sind Didaktisierungsvorschläge anhand solcher Textsorten wie Horoskop, Witz, Buchankündigung und Interview zu finden, die sich an Schüler richten und dementsprechend für die Grundstufe einsetzbar sind. Der dritte Teil ist eher an Germanistikstudierende adressiert und setzt höhere Niveaustufen voraus, weil hier die metasprachliche Reflexion über Phraseologismen im Vordergrund steht (Näheres zu dem Material in Bergerová 2011).

\section{Didaktische Überlegungen}

Es gibt keine allgemein gültigen Rezepte für die erfolgreiche Vermittlung von Phrasemen im DaF-Unterricht. Aber es ist allgemein bekannt, dass die Arbeit mit authentischen oder auch teilweise adaptierten Texten ein guter Weg zur effektiven Vermittlung von Phraseologismen ist. Hervorragend für die Unterrichtsarbeit bieten sich laut Bergerova (2007: 167) kurze Textsorten, z.B. Horoskope, Witze, Filmankündigungen an, weil sie nicht viel Zeit in Anspruch nehmen, meistens wenige Sätze beinhalten und gleichzeitig thematisch abgeschlossene Einheiten darstellen. Im Weiteren wird ein Didaktisierungsvorschlag der Textsorte „Filmankündigung“ auf der Grundlage des Drei- bzw. Vierschritts von Kühn und Lüger präsentiert.

Filmankündigungen erscheinen in Zeitschriften und vor allem auf speziellen Internetseiten, die neue Filme kurz darstellen. Der vorliegende Text stellt einen amerikanischen Film von 2019 dar und heißt „Yesterday“.

Die erste Aufgabe könnte so lauten: Lesen Sie den Text und entscheiden Sie, welche der Behauptungen richtig ist? Wie fühlen sich die Beteiligten wegen der organisierten Tour? Wie wird das im Text formuliert?

In der Musical-Komödie von Oskarpreisträger Danny Boyle sind The Beatles fast vergessen, nur ein Musiker erinnert sich noch an die Hits der Swinging Sixties.

Mit „All You Need Is Love“ haben John Lennon und Paul McCartney einen der größten Hits für die Beatles geschaffen. Doch was wäre, wenn sich niemand mehr daran erinnern würde? Einzig ein unbedeutender britischer Musiker (Himesh Patel) kann sich noch an die legendären Pilzköpfe erinnern. Erst als der SingerSongwriter Ed Sheeran auf den letzten Beatles-Fan aufmerksam wird, beginnt eine grandiose Tour, die allen Beteligten bald über den Kopf wächst (aus: https://www. kino.de/film/yesterday-2019/).

A) Die Beteiligten der Tour sind glücklich.

B) Die Beteiligten der Tour sind überfordert.

Laut der Methode des phraseologischen Drei- bzw. Vierschritts sollen die 
Lernenden den Phraseologismus erkennen und ihn mithilfe vom Kontext entschlüssen können. Semantische Irregularitäten, auf die jeder Lerner bei der Entdeckung eines Phraseologismus bzw. Phrasems trifft, weisen ihn darauf hin, dass die Bedeutung seiner einzelnen Komponenten einen anderen Sinn hat als das Phrasem selbst. In diesem Fall ist die Tour den Beteiligten nicht wirklich über den Kopf gewachsen. Man meint damit etwas anderes, d.h. es gibt eine wörtliche und eine phraseologische (versteckte) Bedeutung dieses Phrasems. An dieser Stelle können Beispielsätze mit dieser Redewendung angeführt werden, um den Lernenden dabei $\mathrm{zu}$ verhelfen, die Bedeutung zu entschlüsseln, indem sie sie mit eigenen Worten umschreiben. Anschließend kann nach litauischen Entsprechungen bzw. Äquivalenten dieser Redewendung gesucht werden, z.B.:

Ich arbeite sehr viel. Die Arbeit wächst mir über den Kopf.

Es wurde ihm einfach zu viel, das Projekt wuchs ihm über den Kopf.

Wenn dir deine Probleme über den Kopf wachsen, melde dich bei mir. Ich helfe dir.

Nachdem die Bedeutung des Phrasems entschlüsselt wurde, können zusätzlich andere Redewendungen mit der Komponente „Kopf“ kennen gelernt werden, z.B.:

Jemandem den Kopf verdrehen: Die gut aussehende Maria hat Daniel den Kopf verdreht, er ist total verliebt in sie.

Den Kopf in den Sand stecken: Er hat schon immer den Kopf in den Sand gesteckt, wenn große Probleme auf ihn zukamen.

Sich um Kopf und Kragen reden: Und Microsofties beten, dass Bill Gates sich vor Gericht nicht wieder um Kopf und Kragen redet.

Als Alternative können auch Redewendungen behandelt werden, die andere menschliche Reaktonen (Glück, Trauer, Wut etc.) bezeichnen, z.B.:

Völlig aus dem Häuschen sein: Sie hat im Lotto gewonnen und ist jetzt völlig aus dem Häuschen.

Große Augen machen: Die Kinder werden große Augen machen, wenn sie unsere Geschenke sehen.

Auf die Palme gehen: Mein Chef würde auf die Palme gehen, wenn ich diesen Auftrag nicht rechtzeitig erledige.

In den beiden Fällen ist es sinnvoll mit Bildern zu arbeiten, die bessere Gedächtnishaftung durch visuelle Assoziationen fördern. Zuerst sollen die Lernenden beschreiben, was sie auf den Bildern sehen und danach können beispielsweise Bilder den entsprechenden Redewendungen und/oder Kommentaren zugeordnet werden. 

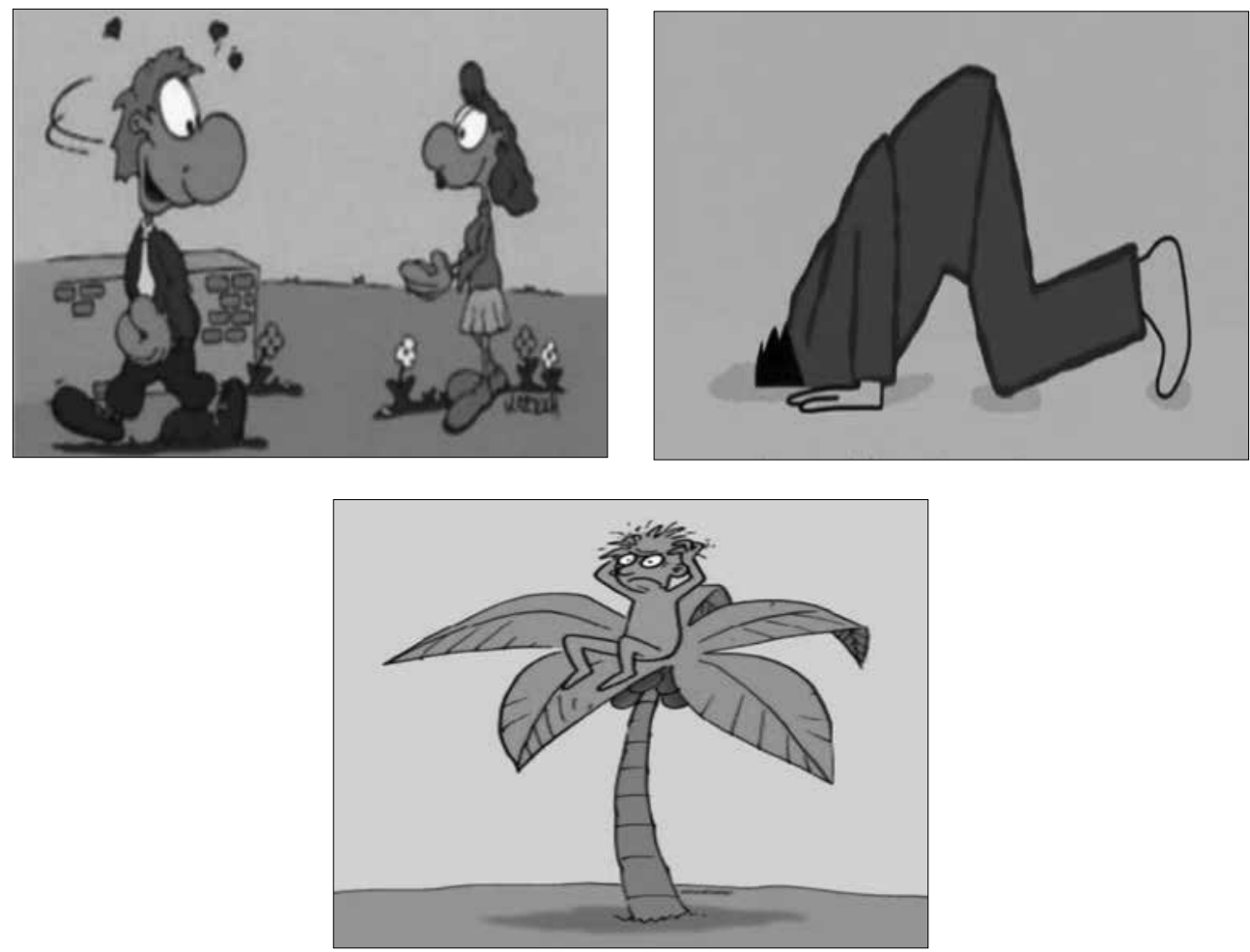

Für den Schritt „Festigen“ können weitere Übungen gemacht werden, wie Multiple-Choice-Aufgaben oder Ergänzungsübungen mit schon gelernten Redewendungen, z.B.:

Ergänzen Sie die fehlenden Teile.

Die ..... voll haben (Nase, Lippe, Stirn) die Palme gehen (auf, in, über)

Den Kopf in den Sand ..... (stecken, legen, hängen)

Große ..... machen (Arme, Augen, Ohren)

Für die Produktionsaufgabe bzw. den Schritt „Verwenden“ könnten Bilder von bekannten Personen verteilt werden, zu denen die Lernenden Kommentare bzw. Dialoge/Monologe formulieren sollen. Dabei werden Redewendungen benutzt, die vorher behandelt wurden. Gegebenfalls können vor allem die fortgeschrittenen Lerner darum gebeten werden, eine eigene Filmankündigung zu schreiben. 

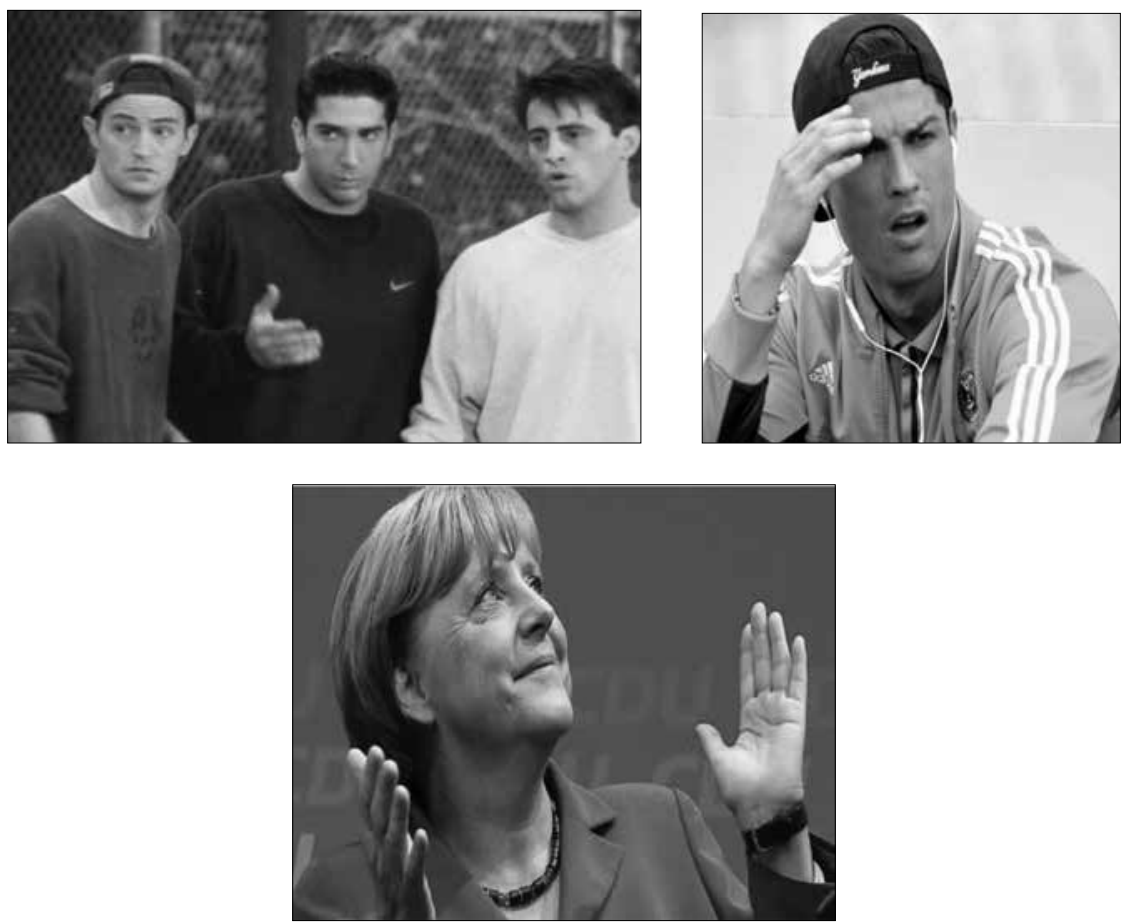

Eine ähnliche Vorgehensweise kann auch mit anderen Textsorten erfolgen, z.B. Buchankündigungen, Horoskope, Lieder, Witze etc.

\section{Fazit:}

Die aktive Beherrschung der Phraseologismen bzw. Phraseme kann den Lernenden in ihrer sprachlichen Kompetenz mehr Sicherheit verleihen. Jedoch wird ihrem Erwerb in den geläufigen DaF-Lehrwerken wenig Aufmerksamkeit geschenkt bzw. sie werden entweder sehr selten oder gar nicht thematisiert. In den meisten DaF-Lehrwerken werden Sprichwörter und Redewendungen behandelt, die nur einen Teilbereich der Phraseme darstellen. Alle anderen Gruppen von phraseologischen Sprachmitteln werden meistens außer Acht gelassen. Aus diesem Grund ist die Gestaltung eigener Lehrmaterialien zur Vermittlung von phraseologischen Ausdrücken vonnöten.

Bei der Erstellung von kreativen Aufgaben ist darauf zu achten, dass die Vermittlung der Phraseme im Fremdsprachenunterricht von der Arbeit mit und an Texten nicht zu trennen ist, weil nur der Kontext dabei hilft, phraseologische Wortverbindungen zu entschlüsseln. Vor allem authentische Texte schließen die Vermittlung ungebräuchlicher Idiome im DaF-Unterricht aus und garantieren ihren gegenwartssprachlichen Gebrauch (Bergerova 2007: 166). Bei der Textauswahl sind vor allem sprachliche Kriterien von Bedeutung, denn sie sollten das Sprachniveau von der Lernenden nicht überschreiten, sie damit nicht demotivieren und nicht über- 
fordern. Dabei soll betont werden, dass auch gewählte Themen bei der Arbeit eine wichtige Rolle spielen.

Phraseme können bei jeder Unterrichtstunde bzw. bei jedem Thema hinzugefügt und behandelt werden. Im vorliegenden Beitrag wurden didaktische Überlegungen zur Phrasemvermittlung im DaF-Unterricht vorgestellt, die sich auf die Methode des phraseodidaktischen Drei- bzw. Vierschritts (Kühn, Lüger) beziehen. Das Anliegen des vorliegenden Beitrags bestand darin, die besten Strategien zur systematischen Einführung der Phraseme in den Unterrichtsprozess zu behandeln. Hier wurde sich vor allem um eine durchaus praktische Annäherung an das Thema bemüht, um das „didaktische Potential“ der Phraseologismen zu zeigen und dabei für die systematische Förderung phraseologischer Kompetenz im DaF-Unterricht zu plädieren.

\section{Literaturverzeichnis}

1. Alenius, M. (2014). Idiome im finnischen DaF-Unterricht. Eine vergleichende Analyse der Lehrwerkreihen Genau und Weitere Wege für die gymnasiale Oberstufe. Tampere: Universität Tampere.

2. Bergerová, H. (2007). Überlegungen zur Phraseologismenvermittlung im DaF-Unterricht. Ein Didaktisierungsvorschlag. In: Aussiger Beiträge 1, S. 163178.

3. Bergerová, H. (2011). Zum Lehren und Lernen von Phraseologismen im DaF-Studium. Überlegungen zu Inhalten und Methoden ihrer Vermittlung anhand eines Unterrichtsmodells. In: Linguistik online 47/3, S. 107-117.

4. Ehrhardt, C. (2014). Idiomatische Kompetenz: Phraseme und Phraseologie im DaFUnterricht. In: gfl-journal 1, S. 1-20.

5. Fleischer, W. (1997). Phraseologie der deutschen Gegenwartsprache. 2. Aufl. Tübingen: Max Niemeyer Verlag.

6. Gündoğdu, M. (2007). Die Behandlung der Phraseologismen im Unterricht Deutsch als Fremd- und Zweitsprache. In: DaZ 2, S. 11-18.

7. Hallsteinsdóttir, E. (2011). Aktuelle Forschungsfragen der deutschsprachigen Phraseodidaktik. In: Linguistik online 47/3, S. 3-32.

8. Hessky, R. 1997. Feste Wendungen - ein heißes Eisen? Einige phraseodidaktische Überlegungen für den DaF-Unterricht". In: Deutsch als Fremdsprache 3, S. 139-143.

9. Kühn, P. (1992). Phraseodidaktik. Entwicklungen, Probleme und Überlegungen für den Muttersprachenunterricht und den Unterricht DaF. In: Fremdsprachen Lehren und Lernen: Zur Theorie und Praxis des Sprachunterrichts an Hochschulen 21. Tübingen: Gunter Narr Verlag, S. 175-177.

10. Lüger, H.H. (1997). Anregungen zur Phraseodidaktik. In: Beiträge zur Fremdsprachenvermittlung 32. Konstanz: Sprachlehrinstitut der Universität Konstanz, S. 69-135. 
11. Niebisch D. et. al. Schritte international neu 1. Deutsch als Fremdsprache. Kurs- und Arbeitsbuch. Ismaning: Hueber Verlag, 2016.

12. Niebisch D. et. al. Schritte international neu 3. Deutsch als Fremdsprache. Kurs- und Arbeitsbuch. Ismaning: Hueber Verlag, 2017.

13. Niebisch D. et. al. Schritte international 3. Kurs- und Arbeitsbuch. 6 Aufl. Ismaning: Hueber Verlag, 2016.

14. Perlmann-Balme M; Schwalb S.; Weers D. 2013. em neu Abschlusskurs, 4. Aufl. Ismaning: Hueber.

15. Wotjak, B. (1996). Redewendungen und Sprichwörter. Ein Buch mit sieben Siegeln? Einführung in den Themenschwerpunkt. In: Fremdsprache Deutsch 15, S. 4-9. 


\title{
PHRASEOLOGICAL UNITS IN GERMAN AS A FOREIGN LANGUAGE TEACHING
}

\author{
Assoc. Prof. Dr. Diana Babušytè \\ Mykolas Romeris University
}

Phraseological units are an inseparable part of daily communication and that makes them an important part of learning the vocabulary of a foreign language. Nevertheless, phrasemes are seldom focused on in modern student books and manuals. This paper advocates a systematic inclusion of phraseology in the teaching and learning German as a foreign language and gives a theoretically well-founded description of teaching German phraseology in German as a foreign language course. It discusses the relevance of phraseology and a stronger integration of phrasemes in language teaching and didactics as well as overviews the learning materials for teaching phrasemes.

The lack of adequate learning materials and exercises concerning phraseology teaching demands a lot of creativity from teachers. The most famous model of teaching phrasems is introduced by Kühn and Lüger. It is based on the three-four steps ("phraseodidaktischer Drei- bzw. Vierschritt") of teaching phraseology and emphasizes that phraseological units should be taught and acquired on the basis of authentic texts. Linguistic criteria for choosing a text are of particular importance because they should not exceed the language level of the students and, therefore, not demotivate or overwhelm them. Also, selected topics play an important role in the teaching process. This paper presents a didactic proposal based on this model using the text type "description of the film". The first step ("Erkennen" recognize) is the identification of phrasemes in a text. Learners have to be able to realize that a construction of words is meant in the indirect sense. The second step ("entschlüsseln" decode) includes the process of understanding the meaning of a phraseological unit in the context. The third step ("festigen" - consolidate) is the process of acquiring phrasemes with the help of suitable exercises, and the fourth step ("verwenden" - apply) is concentrated on the use of phrasemes.

Generally, phraseological units can be included in every lesson or topic. The suggestions for teaching phrasemes that have been made in this paper represent an exemplary approach to the phraseology in learning German vocabulary. The aim of this paper is to emphasize the relevance of phraseology in teaching foreign languages and to encourage teachers for this challenging but rewarding area of language learning, especially at the basic level, and to motivate them to use their own creative materials for teaching German phrasemes. 


\section{AUTORIAUS LYDRAŠTIS}

Autoriaus vardas, pavardė: Diana Babušytė

Mokslo laipsnis ir vardas: humanitarinių mokslų daktarè

Darbo vieta ir pareigos: Mykolo Romerio universiteto profesore

Autoriaus mokslinių interesų sritys: dabartinė vokiečių kalba, kontrastyvinè lingvistika, eurolingvistika, kalbų tipologija, svetimųų kalbų dèstymo didaktika

Telefonas ir el. pašto adresas: 864590727; diana.babusyte@mruni.eu

\section{AUTHOR'S COVER LETTER}

Author's name and surname: Diana Babušytė

Academic degree and name: Doctor of Arts

Workplace and position: Mykolas Romeris University Professor, Doctor

Author's research interests: modern German, contrastive linguistics, eurolinguistics, linguistic typology, foreign language teaching methods

Telephone and e-mail address: +370 64590 727; diana.babusyte@mruni.eu 